\title{
FIXED POINTS OF POINTWISE ALMOST PERIODIC HOMEOMORPHISMS ON THE TWO-SPHERE
}

\author{
BY
}

\author{
W. K. MASON(1)
}

ABSTRACT. A homeomorphism $f$ of the two-sphere $s^{2}$ onto itself is defined to be pointwise almost periodic (p.a.p.) if the collection of orbit closures forms a decomposition of $s^{2}$. It is shown that if $f: s^{2} \rightarrow s^{2}$ is p.a.p. and orientation-reversing then the set of fixed points of $f$ is either empty or a simple closed curve; if $f: s^{2} \rightarrow s^{2}$ is p.a.p. orientation-preserving and has a finite number of fixed points, then $f$ is shown to have exactly two fixed points.

1. Introduction. Every periodic mapping $f$ of the two-sphere $S^{2}$ to itself is topologically equivalent either to the identity, to a rotation, a reflection, or to a rotation followed by a reflection ([5] and [9]). Thus, the set of fixed points of $f$ is either empty or an $i$-sphere, $0 \leqslant i \leqslant 2$. If $f$ satisfies the weaker condition of being almost periodic (equivalent to having equicontinuous iterates) or the still weaker condition of being weakly almost periodic (the collection of orbit closures forms a continuous decomposition of $S^{2}$ ), the fixed point set is again either empty or an $i$-sphere, $0 \leqslant i \leqslant 2$ ([11] and [12]).

In this paper we study the fixed point sets of pointwise almost periodic (p.a.p.) homeomorphisms on $S^{2}$ (the collection of orbit closures forms a decomposition of $S^{2}$ ). In the orientation-reversing case the set of fixed points must still be either empty or a 1-sphere (Theorem 6). In the orientation-preserving case, on the other hand, there may be a continuum of fixed points together with any finite or countable number of isolated fixed points (see §6). However, if there are only a finite number of fixed points in the orientation-preserving case, then there must be exactly two (Theorem 7).

The main theorems of this paper are contained in $\S \S 5$ and $6 . \S 3$ gives a summary of the results in the theory of prime ends which we use to prove the main lemma in $\S 4$.

2. Definitions and notation. If $A$ is a subset of a space $X, \mathrm{Cl}(A)$ and $\operatorname{Bd}(A)$ denote, respectively, the closure and boundary of $A . A$ is nondegenerate if $A$ is not a single point. If $f: X \rightarrow X$ is a map, then $f \mid A$ denotes the restriction

Received by the editors October 9, 1973 and, in revised form, March 11, 1974.

AMS (MOS) subject classifications (1970). Primary 54H20, 55C20, 57A05; Secondary $54 \mathrm{H} 25$.

Key words and phrases. Pointwise almost periodic transformation, recurrent point, prime ends, periodic transformation.

(1) Research partially supported by NSF GP-33943. 
of $f$ to $A$. Homeomorphisms will always be onto.

A domain is a connected open set. If $U$ is a domain in $S^{2}$ and $M$ is a subset of a component $R$ of $S^{2}-\mathrm{Cl}(U)$, then $\mathrm{Bd}(R)$ is the outer boundary of $U$ with respect to $M$.

If $f: X \rightarrow X$ is a homeomorphism and $A \subset X$, then orbit( $A$ ), the orbit of $A$, is the union of the set of iterates $f^{n}(A), n=0, \pm 1, \pm 2, \cdots,\left(f^{0}=\right.$ Id). The set of period two points of $f$ is the set $\left\{x \in X: f^{2}(x)=x\right\}$. Thus, the set of period two points includes the fixed points of $f$.

$f: X \rightarrow X$ is a recurrent homeomorphism if, given any $x \in X$ and any neighborhood $U$ of $x$, there is a positive integer $n$ and a negative integer $m$ such that $\left\{f^{n}(x), f^{m}(x)\right\} \subset U[8,10.18$, p. 83] .

$f: X \rightarrow X$ is a pointwise almost periodic (p.a.p.) homeomorphisms if given any $x \in X$ and any neighborhood $U$ of $x$, there is a finite set $K$ of integers such that the orbit of $x$ is contained in the union of the sets $f^{n}(U), n \in K$ $\left[8,4.02\right.$, p. 31]. If $X$ is a locally compact $T^{2}$ space, an equivalent definition is: $f: X \rightarrow X$ is p.a.p. if the collection $\{\mathrm{Cl}($ orbit $(x)$ ): $x \in X$ \} forms a decomposition of $X[8,4.10$, p. 32]. Note that if a homeomorphism is p.a.p. then it is recurrent.

If $U$ is a domain in $S^{2}$ with a nondegenerate boundary, then a crosscut of $U$ is an open arc in $U$ whose closure is an arc which intersects $\operatorname{Bd}(U)$ in two points. An endcut of $U$ is a half-open arc in $U$ whose closure is an arc which intersects $\operatorname{Bd}(U)$ in one point. If $A$ is a crosscut or an endcut of $U$, then a subendcut of $A$ is an endcut of $U$ which is contained in $A$.

3. Prime ends. This section contains the results from the theory of prime ends which we use in the next two sections. See also [12, §3] or [15].

Let $U$ be a simply connected domain in $S^{2}$ with a nondegenerate boundary. A $C$-transformation of $U$ onto the open unit disk $D$ is a homeomorphism $T: U \rightarrow D$ such that the image of any crosscut of $U$ is a crosscut of $D$, and the endpoints of such images of crosscuts of $U$ are dense in the unit circle. $C$-transformations always exist [15, Appendix 2].

A collection of crosscuts $Q_{1}, Q_{2}, \cdots$, of the simply-connected domain $U$ is a chain if (a) the arcs $\mathrm{Cl}\left(Q_{1}\right), \mathrm{Cl}\left(Q_{2}\right), \cdots$, are pairwise disjoint; (b) $Q_{n}$ separates $Q_{n-1}$ from $Q_{n+1}$ in $U$; (c) there is a point on $\operatorname{Bd}(U)$ whose greatest distance from $Q_{n}$ approaches 0 as $n \rightarrow \infty$. Corresponding to each $Q_{n}$ there is a domain $U_{n}$ of $U-Q_{n}$ containing $Q_{n+1}$. Note $U_{1} \supset U_{2} \supset \cdots$.

If $\left\{Q_{i}\right\},\left\{R_{i}\right\}$ are chains of crosscuts and $\left\{U_{i}\right\},\left\{H_{i}\right\}$ are their respective corresponding domains, then $\left\{Q_{i}\right\},\left\{R_{i}\right\}$ are equivalent chains if for every $n$ there is an $m$ such that $H_{m} \subset U_{n}$ and $U_{m} \subset H_{n}$. Equivalent chains are said to define the same prime end. Thus, a prime end of $U$ is an equivalence class of chains of $U$. 
If $Q_{1}, Q_{2}, \cdots$ is a chain of crosscuts in $U$, then their images $T\left(Q_{1}\right)$, $T\left(Q_{2}\right), \cdots$ under the $C$-transformation $T: U \rightarrow D$ is a chain in $D$. If $\left\{Q_{i}\right\},\left\{R_{i}\right\}$ are equivalent chains in $U$, then $\left\{T\left(Q_{i}\right)\right\},\left\{T\left(R_{i}\right)\right\}$ are equivalent chains in $D$ and converge to the same point on the boundary of $D$. Thus, $T$ sets up a 1-1 correspondence between prime ends of $U$ and points on the unit circle.

If $Q_{1}, Q_{2}, \cdots$ is a chain defining the prime end $E$ and $U_{1}, U_{2}, \cdots$ are the corresponding domains of the chain, then the impression of $E$ is the set $\bigcap_{i=1}^{\infty} \mathrm{Cl}\left(U_{i}\right)$. The impression of $E$ is easily seen to be independent of which defining chain is used, Note that Impression $(E) \subset \operatorname{Bd}(U)$ and that distinct prime ends may have identical impressions.

Suppose $U$ is a simply connected domain, $T: U \rightarrow D$ is a $C$-transformation, $E$ is a prime end of $U$, and $e$ is the point on the unit circle corresponding to $E$ under $T$. A half-open arc $B$ in $U$ defines the prime end $E$ if $T(B)$ is an endcut in $D$ with $e$ as a limit point. Among the half-open arcs defining the prime end $E$ there is one $A$ such that $\mathrm{Cl}(A)-A$ is minimal (contained in $\mathrm{Cl}(B)-B$ for every half-open arc $B$ defining $E$ ). This minimal set is the set of principal points of $E$ (for an alternate definition of principal point see $[12, \S 3])$. Note that a prime end defined by an endcut has just one principal point.

Given a homeomorphism $f: \mathrm{Cl}(U) \rightarrow \mathrm{Cl}(U)$, with $f(U)=U$, and a $C$ transformation $T: U \rightarrow D$, it follows that $T f T^{-1}: D \rightarrow D$ is a $C$-transformation which may be extended to a homeomorphism $h$ of the closed unit disk onto itself $[15,4.10$, p. $6 ; A 1.7$, p. 27]. If $E$ is a prime end of $U, e$ is the point on the unit circle corresponding to $E$ under $T$, and $h(e)=e$, then $E$ is a fixed prime end of $f$. If $G$ is another prime end of $U, p$ is the point on the unit circle corresponding to $G$ under $T$, and $p$ converges to $e$ under positive iterates of $h$, then we say the prime end $G$ converges to the prime end $E$ under positive iterates of $f$. These last two definitions are independent of the choice of the $C$-transformation $T$.

The reader unfamiliar with prime ends might attempt to show as an exercise that if $K$ is a pseudo-arc [1] and $E$ is a prime end of $S^{2}-K$ then Impression $(E)=K$; also, there exists a prime end $E$ of $S^{2}-K$ such that every point of $K$ is a principal point of $E$. Completing this exercise, however, is not necessary for understanding the rest of the paper.

4. The Main lemma. Preliminary remark: In the proof of Lemma 1 below, various crosscuts and endcuts are constructed in a domain $U$. Our diagrams, however, will always show the images in the open unit disk of these crosscuts and endcuts under a $C$-transformation. To avoid clumsy notation the crosscuts and endcuts will be denoted with the same letters as their $C$. images in the diagrams. 
Lemma 1. Suppose $f: S^{2} \rightarrow S^{2}$ is an orientation-preserving homeomorphism and $U$ is an invariant, simply-connected domain with nondegenerate boundary. Suppose $A$ and $B$ are endcuts of $U$ such that (1) the prime end $E$ of $U$ which is defined by $A$ is fixed under $f$, and (2) the prime end $F$ of $U$ which is defined by $B$ is distinct from $E$ but converges to $E$ under positive iterates of $f$. Then $f$ is not recurrent on $\operatorname{Bd}(U)$.

Proof. Let $b$ be the point $\mathrm{Cl}(B) \cap \operatorname{Bd}(U)$.

Case $1 . b$ is not in the impression of the prime end $E$. Then let $V$ be a neighborhood (in $S^{2}$ ) of $b$ whose closure misses Impression $(E)$. Let $U_{1} \supset U_{2} \supset \cdots$ be a sequence of subdomains of $U$ such that Impression $(E)=$ $\bigcap_{i=1}^{\infty} \mathrm{Cl}\left(U_{i}\right)$. Then for some $n, U_{n} \cap V=\varnothing$. If $f$ were recurrent at $b$, then subendcuts of infinitely many positive iterates of $B$ would be contained in $V$, and thus would miss $U_{n}$. Then the prime end $F$ would not converge to the prime end $E$.

Case 2. $f$ is periodic at $b$, with least period $n$. If $f(b)=b, n=1$, let $Y_{1}$ be an open arc in $U$ such that $\mathrm{Cl}\left(Y_{1}\right)$ is a simple closed curve and $Y_{1}$ contains a subendcut of $B$ and a subendcut of $f(B)$. If $f^{n}(b)=b, n>1$, let $Y_{1}, \cdots, Y_{n}$ be a set of pairwise disjoint crosscuts of $U$ such that each $Y_{i}$ contains a subendcut defining the same prime end as $f^{i-1}(B)$ and a subendcut defining the same prime end as $f^{i}(B), 1 \leqslant i \leqslant n$. Note that $\mathrm{Cl}\left(Y_{n}\right) \cap$ $\mathrm{Cl}\left(Y_{1}\right)=\{b\}=\left\{f^{n}(b)\right\}$. In both cases, $\mathrm{Cl}\left(Y_{1}\right) \cup \cdots \cup \mathrm{Cl}\left(Y_{n}\right)$ forms a simple closed curve $J$ such that $J$ separates a subendcut of $A$ from the closure of some endcut $N$ of $U$ (see Figure 1 for a sketch of the $C$-images of $Y_{1}, \cdots$, $\left.Y_{n}, A, N\right)$. Note that $\mathrm{Bd}(U) \cap \mathrm{Cl}(N)$ cannot lie in Impression $(E)$.

Now, if $J$ is the arc in the unit circle bounded by the endpoints of the $C$ images of $A$ and $B$ and containing the endpoint of the $C$-image of $f(B)$, and if $h$ is the (orientation-preserving) homeomorphism of the closed unit disk associated with $f$ (see definition of convergent prime end, §3), then $h\left(J^{\prime}\right) \subset$ $J^{\prime}$. The endpoint of the $C$-image of $N$ is in $J^{\prime}$ and thus converges to the fixed endpoint of $\boldsymbol{J}^{\prime}$ under positive iterates of $h$.

But then the prime end determined by $N$ converges to the prime end $E$, and $\mathrm{Bd}(U) \cap \mathrm{Cl}(N)$ cannot lie in Impression $(E)$. Hence, by Case 1, $\operatorname{Bd}(U) \cap \mathrm{Cl}(N)$ is not a recurrent point of $f$.

Case 3. $b$ is in the impression of $E$, but is not a periodic point. We suppose $f$ recurrent on $\operatorname{Bd}(U)$ and derive a contradiction.

Our plan is to construct a simple closed curve $J$, made up of crosscuts of $U$ plus an arc $Y$, such that $J$ separates the endcut $A$ and some point of Impression $(E)$, (to construct $J$ we may have to modify $f$ on some subdisks of $U$ ), then to obtain a certain subcontinuum $L$ of Impression $(E)$ such that $L \cap Y \neq \varnothing$ but $L$ misses one component of $S^{2}-J$, and finally to obtain 




FIGURE 1

the contradiction that $f(L)=L$ but $f(L)$ must intersect both components of $S^{2}-J$. We now proceed with this plan.

Since $E$ is a fixed prime end we may assume $f=$ Id on $A$. For, there is an open disk $Z \subset U$ and a subendcut $A^{\prime}$ of $A$ such that $\mathrm{Cl}(Z) \cap \mathrm{Bd}(U)=$ $\mathrm{Cl}(A) \cap \operatorname{Bd}(U)$, and $A^{\prime} \cup f\left(A^{\prime}\right) \subset Z$. Then replace $A$ by $A^{\prime}$ and $f$ by $f$ followed by a homeomorphism which is the identity outside $Z$ and which is equal to $f^{-1}$ on $f\left(A^{\prime}\right)$.

Choose a crosscut $Q$ of $U-A$ such that (a) $Q$ has one endpoint on $A$ and the other on $\operatorname{Bd}(U)$, (b) there is a positive integer $n$ such that $Q$ separates $f^{-1}(B) \cup B \cup f(B)$ from some subendcut of $f^{n}(B)$ in $U-A$, (c) $\mathrm{Cl}(Q)$ is disjoint from $\mathrm{Cl}\left(f^{-1}(B) \cup B \cup f(B)\right)$ and from $f^{n}(b)$. The existence of $Q$ follows from the fact that the prime end defined by $B$ converges to the prime end defined by $A$.

Next, choose a crosscut $X$ in $U$ such that (d) the endpoints of $X$ are $b$ and $f(b)$, (e) $X$ contains a subendcut defining the prime end, $F$, (f) the crosscuts $f^{-1}(X), X, f(X), \cdots, f^{n}(X)$ form a pairwise disjoint collection (here, $n$ is the integer mentioned in (b) of the preceding paragraph), and (g) $Q$ separates $f^{-1}(X)$ from $f^{n}(X)$ in $U-A$. See Figure 2 for a sketch of $C$-images.

The existence of $X$ follows from the facts that $f(b) \neq b$ and that $F$ converges to $E$.

Next, choose an open (in $S^{2}$ ) neighborhood $O$ of $b$ such that

$\mathrm{Cl}(O)$ is a 2-cell;

$\mathrm{Cl}(O) \cap f(\mathrm{Cl}(O))=\varnothing$;

$[A \cup \mathrm{Cl}[(Q)] \cap[\mathrm{Cl}(O) \cup f(\mathrm{Cl}(O))]=\varnothing$;

$\mathrm{Cl}(O) \cap\left[\mathrm{Cl}(X) \cup f^{-1}(\mathrm{Cl}(X))\right]$ is an arc; and

$\mathrm{Cl}(O) \cap f^{i}(X)=\varnothing$ for $i=1,2, \cdots, n$. 


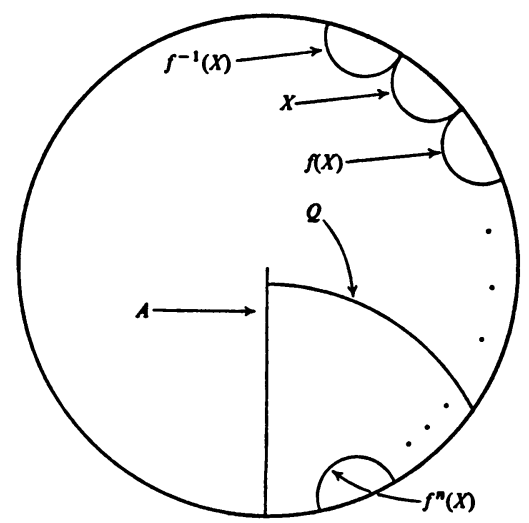

FIGURE 2

Claim. There is a homeomorphism $k: S^{2} \rightarrow S^{2}$ such that:

(1) $k=f$ on some neighborhood of $\operatorname{Bd}(U) \cup \mathrm{Cl}(O) \cup A$,

(2) $k^{i}(X)=f^{i}(X)$ for $i=-1,0,1, \cdots, n$, (3) for some integer $m>n$, $k^{-1}(X), X, k(X), \cdots, k^{m}(X)$ is a pairwise disjoint collection, $k^{i}(X) \cap \mathrm{Cl}(O)=$ $\varnothing, n \leqslant i<m, \quad k^{m}(X) \cap \mathrm{Cl}(O) \neq \varnothing$, and $Q$ separates $k^{-1}(X)$ and $k^{i}(X)$ in $U-A, n \leqslant i \leqslant m$.

Proof of claim. We construct $k$ by modifying $f$ on various subdisks of $U$.

Suppose $f^{n+1}(X) \cap Q \neq \varnothing$. Let $W$ be the component of $U-$ $\left(f^{n}(X) \cup f^{-1}(Q)\right)$ such that $\operatorname{Bd}(W)$ contains two disjoint subendcuts of $f^{n}(X)$. Let $N$ be a crosscut of $W$ such that $\operatorname{Cl}(N) \cap \operatorname{Bd}(U)=\varnothing$, the endpoints of $N$ separate $f^{n}(b)$ and $f^{n+1}(b)$ from $f^{-1}(Q) \cap f^{n}(X)$ in $\mathrm{Cl}\left(f^{n}(X)\right)$, and $N$ is contained in a small neighborhood of $f^{n}(X) \cup f^{-1}(Q)$. Since $f^{n}(X) \cap \mathrm{Cl}(O)=\varnothing$ and $\mathrm{Cl}(Q) \cap f(\mathrm{Cl}(O))=\varnothing$, we may choose $N$ so that $\mathrm{Cl}(N) \cap \mathrm{Cl}(O)=\varnothing$, (see Figure 3 for $C$-images).

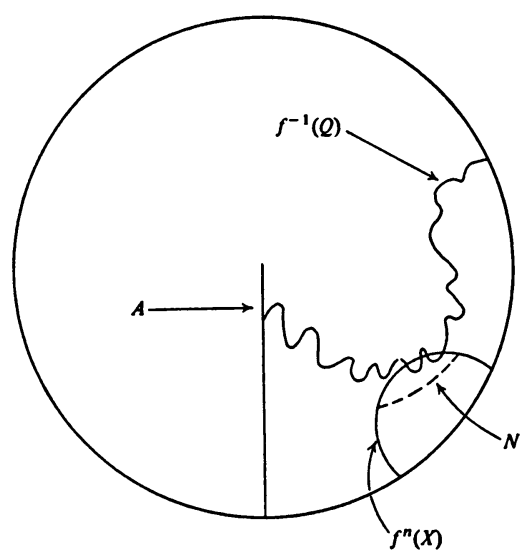

Figure 3 
Let $Z \subset U$ be the disk bounded by $N$ and the subarc of $f^{n}(X)$ cut off by the endpoints of $N$. Then $Z$ misses $\mathrm{Cl}(O)$ since $\mathrm{Bd}(Z)$ misses $\mathrm{Cl}(O)$. Let $Z_{1}$ be a small neighborhood of $Z$ such that $\mathrm{Cl}\left(Z_{1}\right)$ is a subdisk of $U$, $\mathrm{Cl}\left(Z_{1}\right) \cap \mathrm{Cl}(O)=\varnothing, \mathrm{Cl}\left(Z_{1}\right) \cap A=\varnothing$, and $Z_{1} \cap f^{i}(X)=\varnothing, i=-1,0,1, \cdots$, $n-1$. Let $g: S^{2} \rightarrow S^{2}$ be a homeomorphism such that $g=$ Id outside $Z_{1}$, $g=$ Id on $f^{n}(X)-Z$, and $g(\operatorname{Bd}(Z)-N)=\mathrm{Cl}(N)$. Finally, let $h=f g$.

Note that (1) $h=f$ on some neighborhood of $\operatorname{Bd}(U) \cup \mathrm{Cl}(O) \cup A$, (2) $h^{n+1}(X) \cap Q=\varnothing$, and (3) $\left\{h^{-1}(X), X, h(X), \cdots, h^{n+1}(X)\right\}=\left\{f^{-1}(X)\right.$, $\left.X, f(X), \cdots, f^{n}(X)\right\} \cup\left\{h^{n+1}(X)\right\}$ is a pairwise disjoint collection. (For, $n^{n+1}(X)$ can intersect the preceding images of $X$ only in $f^{-1}(X)$; otherwise, the preceding images would not he disjoint. But $Q$ separates $h^{n+1}(X)$ and $f^{-1}(X)$.)

If $\mathrm{Cl}\left(h^{n+1}(X)\right) \cap \mathrm{Cl}(O) \neq \varnothing$, then $h$ is the homeomorphism we seek. If $\mathrm{Cl}\left(h^{n+1}(X)\right) \cap \mathrm{Cl}(O)=\varnothing$, we repeat the above process, modifying $h$ to add another image of $X$ to our collection. This process must finally terminate, however, because we are assuming that $f$ is recurrent at $b$, and $b \in O$, so eventually some image of $X$ will intersect $\mathrm{Cl}(O)$. This completes the proof of our claim.

To simplify notation let us assume that $f$ requires no modification, that $f^{n+1}(X) \cap Q=\varnothing$ and $\mathrm{Cl}\left(f^{n+1}(X)\right) \cap \mathrm{Cl}(O) \neq \varnothing$.

Let $P$ be the arc $\left[\mathrm{Cl}(X) \cup f^{-1}(\mathrm{Cl}(X))\right] \cap \mathrm{Cl}(O)$. We may assume that $O$ was chosen small enough so that one of the components $V$ of $\mathrm{Cl}(O)-P$ is contained in $U-(A \cup Q)$. Then we must have $\mathrm{Cl}\left(f^{n+1}(X)\right) \cap \mathrm{Cl}(O)$ contained in $\mathrm{Cl}(O)-V$. Let $Y$ be an arc in $\mathrm{Cl}(O)-V$ from $b\left(=\mathrm{Cl}(X) \cap f^{-1}(\mathrm{Cl}(X))\right)$ to a point of $\mathrm{Cl}\left(f^{n+1}(X)\right)$ such that, except for its endpoints, $Y$ misses $\operatorname{Cl}\left(f^{-1}(X) \cup X \cup f(X) \cup\right.$ $\left.\cdots \cup f^{n+1}(X)\right)$. Then from the set $Y \cup \operatorname{Cl}\left(X \cup f(X) \cup \cdots \cup f^{n+1}(X)\right)$ we may form a simple closed curve $J$.

Note that $f(Y-\{b\})$ does not intersect $J$, because $Y \subset \mathrm{Cl}(O)$ and $f(\mathrm{Cl}(O)) \cap \mathrm{Cl}(O)=\varnothing$ (this is the reason for never modifying $f$ on $\mathrm{Cl}(O)$ ).

Also, note that $\mathrm{Cl}(A)$ and $f(Y-\{b\})$ are in different components of $S^{2}-J$. For, we may obtain an arc $C$ from $A$ to $f(b)$, which does not intersect $J-\{f(b)\}$, by starting at $A$ and traveling along close to $Q$ and then close to $f^{n-1}(X) \cup f^{n-2}(X) \cup \cdots \cup f(X)$ until we hit $f(V)$, and then traveling through $f(V)$ up to $f(b)$. Then $C \cup f(Y)$ is an arc which intersects $J$ in the piercing point $f(b)$, and thus its endpoints must lie in different components of $S^{2}-J$.

Let $H$ be the component of $S^{2}-J$ containing the endcut $A$. We want to construct a subcontinuum $L$ of $\operatorname{Bd}(U)$ such that $L \subset \mathrm{Cl}(H), L \cap Y \neq \varnothing$, and $f(L)=L$. This will yield a contradiction.

Choose a chain $R_{1}, R_{2}, \cdots$ of crosscuts of $U$ defining $E$, with corresponding domains $U_{1} \supset U_{2} \supset \cdots$, such that

$$
U_{1} \cap\left(f^{-1}(X) \cup X \cup \cdots \cup f^{n+1}(X)\right)=\varnothing \text { and } U_{1} \cap f(V)=\varnothing .
$$


Note that $f(b) \in \mathrm{Cl}\left(U_{i}\right), i=1,2, \cdots$, since $b \in$ Impression $(E)$, and $E$ is a fixed prime end. Let $A_{1}$ be an arc in $U_{1}$ from $A$ to a point very close to $f(b)$. Since $U_{1} \cap f(V)=\varnothing$, we must have $A_{1} \cap J \neq \varnothing$, hence $A_{1} \cap Y \neq$ $\varnothing$. Let $B_{1}$ be the subarc of $A_{1}$ from $A \cap A_{1}$ to the first point at which $A_{1}$ intersects $Y$. Then $B_{1} \subset \mathrm{Cl}(H)$.

Repeating this procedure, we may construct a sequence $B_{1}, B_{2}, \cdots$ of disjoint arcs of $U$ such that:

(1) $B_{i}$ intersects $A$ and $Y$,

(2) $B_{i} \subset \mathrm{Cl}(H)$,

(3) $B_{i} \subset U_{m(i)}$, for some $m(i) \geqslant i$.

We may assume all $B_{i}$ 's lie on the "same side" of $A$ ( $A \cup Q$ does not separate any $B_{i}-A$ from any $B_{j}-A$ in $U$ ) and that the $B_{i}$ 's converge to a subcontinuum $L$ of $\operatorname{Bd}(U)$. (See Figure 4 for $C$-images.)

But then we may choose a half-open arc $T \subset U$ in a small neighborhood of $A \cup B_{1} \cup B_{2} \cup \cdots$ such that $T \cap A=\varnothing, T$ also defines the prime end $E$, and $\mathrm{Cl}(T)-T=L$. Since $f$ is orientation-preserving, both $T$ and $f(T)$ lie on the same side of $A$. Thus, by [15, 2.2, p. 2] or [14, 3.38, p. 321], either $L \subset f(L)$ or $f(L) \subset L$. But we are assuming that $f$ is recurrent on $\operatorname{Bd}(U)$ so we must have $f(L)=L \quad[16,4.12$, p. 247]

Note also that $L \subset \mathrm{Cl}(H)$ and $L \cap Y \neq \varnothing$.

This easily yields a contradiction, for if $L \cap(Y-\{b\}) \neq \varnothing$, then, since $f(Y-\{b\}) \subset S^{2}-\mathrm{Cl}(H)$, we cannot have $f(L)=L$; and, if $b \in L$, then $f(L)=$ $L$ is a continuum containing $f(b)$, but every nondegenerate subcontinuum of $\operatorname{Bd}(U)$ containing $f(b)$ must intersect $S^{2}-\mathrm{Cl}(H)$, since $f(V) \cap \operatorname{Bd}(U)=\varnothing$.

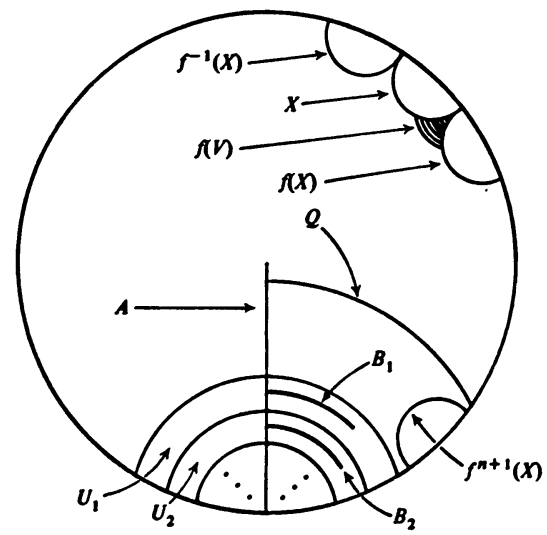

FIGURE 4

This final contradiction establishes Lemma 1. 


\section{Fixed points of orientation-reversing p.a.p. homeomorphisms.}

LEMMA 2. Let $f: X \rightarrow X$ be a p.a.p. homeomorphism of the complete metric space $X, K$ a compact invariant subset of $X$, and $U$ an open subset of $X$ such that $U-K \neq \varnothing$. Then there is an open subset $V$ of $U$ such that the orbit of $V$ misses some neighborhood of $K$.

Proof. For each positive integer $n$, let $R(n)=\{x \in U-K$ : for some integer $\left.m, \operatorname{dist}\left(f^{m}(x), K\right)<1 / n\right\} . U-K$ is complete and each $R(n)$ is open in $U-K$. Therefore, some $R(n)$ is not dense in $U-K$; otherwise, $\bigcap_{n=1}^{\infty} R(n)$ is not empty by the Baire category theorem, but $f$ cannot be p.a.p. at points of $\bigcap_{n=1}^{\infty} R(n)$. Hence, for some positive integer $m$, there is an open set $V$ in $U-K$ which misses $R(m) . V$ is the required open set, and the proof is complete.

LEMma 3. Suppose $f: S^{2} \rightarrow S^{2}$ is an orientation-reversing p.a.p. homeomorphism, $p$ is a fixed point of $f$, and $Y$ is the component of the set of period two points such that $p \in Y$. Then $Y$ is nondegenerate.

Proof. Assume $Y=\{p\}$. We shall establish a contradiction.

Denote by $P(2, f)$ the set of period two points of $f$.

Claim 1. There is an orientation-reversing, p.a.p. self-homeomorphism of $S^{2}$ whose set of period two points is totally disconnected and contains a fixed point.

Proof of Claim 1. Let $G$ be the decomposition of $S^{2}$ whose elements are the points of $S^{2}-P(2, f)$ and the components of $P(2, f) . G$ is upper semicontinuous [7, p. 137], hence the decomposition space $S^{2} / G$ is a cactoid $\left[16,(2.2)^{\prime}\right.$, p. 172]. It is easily seen that the induced map $g=\pi f \pi^{-1}: S^{2} / G \rightarrow S^{2} / G$ (where $\pi: S^{2} \rightarrow S^{2} / G$ is the decomposition map) is a p.a.p. homeomorphism, and $P(2, g)=\pi(P(2, f))$. Thus, $P(2, g)$ is totally disconnected. Now, $\pi(p)$ is not a cut point of $S^{2} / G$, since $\pi^{-1}(\pi(p))=p$, so $\pi(p)$ is either an endpoint of $S^{2} / G$ or is contained in a true cyclic element of $S^{2} / G,[16$, p. 66]. If $M$ is a true cyclic element (2-sphere) containing $\pi(p)$, then $g(\pi(p))=\pi(p)$, so $g(M)=M$, and $g \mid M$ is the required homeomorphism (it is clear that $g$ must be orientation. reversing).

If $\pi(p)$ is an endpoint, then there is a cutpoint $\pi(q)$ fixed by $g[16,4.22$, p. 247]. If $C(\pi(q), \pi(p))$ is the cyclic chain [16, p. 71] of $S^{2} / G$ from $\pi(q)$ to $\pi(p)$, then every cyclic element of $C(\pi(q), \pi(p))$ is invariant under $g$ [16, 4.3, p. 248]. Let $M$ be any true cyclic element of $C(\pi(q), \pi(p))$. Then $M$ is a 2-sphere and $g(M)=M . M$ contains a point which separates $\pi(q)$ and $\pi(p)$ in $S^{2} / G[16,5.2$, p. 71$]$, and this point must be a fixed point of $g[16,4.21$, p. 247]. Thus, $g \mid M$ is the required orientation-reversing homeomorphism.

Claim 1 has been established. 
By Claim 1 we amy assume without loss of generality that $P(2, f)$ is totally disconnected.

Let $x, y$ be points of $S^{2}-\{p\}$ such that $f(x)=y$, and $f(y)=x(x=$ $y$ is allowed). The existence of $x, y$ follows from the fact that if $f^{2} \mid S^{2}-\{p\}$ were fixed point free, then $S^{2}-\{p\}$ would contain a point converging to $p$ under positive iterates of $f^{2} \quad[2$, Theorem $8, \mathrm{p} .45]$ (or see Theorem 7 of the present paper). Let $K$ be a continuum in $S^{2}$ which is invariant under $f$, which contains $\{p, x, y\}$ and which is minimal with respect to containing $\{p, x, y\}$ and being closed, connected, and invariant. By Lemma 2, there is a set $U_{1} \subset K$, open in $K$, such that the orbit of $U_{1}$ misses a neighborhood of $P(2, f) \cap K$. Let $A_{1}$ be the component of $K-\operatorname{orbit}\left(U_{1}\right)$ containing $p$. Note that $A_{1}$ is invariant and nondegenerate. Since $K$ is minimal, $A_{1}$ cannot contain $x$ or $y$. Let $D_{1}$ be the component of $S^{2}-A_{1}$ containing $x$.

Claim 2. $f\left(D_{1}\right) \cap D_{1}=\varnothing$.

Proof of Claim 2. Suppose $f\left(D_{1}\right) \cap D_{1} \neq \varnothing$. Then $D_{1}$ is a simply-connected, invariant domain with a nondegenerate boundary. We note that $D_{1}$ has a prime end which is fixed under $f$. For, let $T$ be a $C$-transformation of $D_{1}$ onto the open unit disk, and extend $T f T^{-1}$ to an orientation-reversing homeomorphism $h$ of the closed unit disk onto itself. Then $h$ must have two fixed points on the unit circle, and these two fixed points correspond to fixed prime ends of $f$.

But then the orientation-preserving homeomorphism $f^{2}$ must also have a fixed prime end in $D_{1}$. Hence, every prime end of $D_{1}$ is either fixed under $f^{2}$ or converges to a fixed prime end under positive iterates of $f^{2}$ [3, Lemma 14].

Since $P(2, f)$ is totally disconnected and closed, there is an endcut $B$ in $D_{1}$ such that $\mathrm{Cl}(B) \cap \mathrm{Bd}\left(D_{1}\right)$ is not in $P(2, f)$. Thus, if $F$ is the prime end defined by $B$, the principal point of $F$ is not fixed under $f^{2}$, and so $F$ is not a fixed prime end of $f^{2}$ [12, Lemma 1]. Thus, $F$ converges under positive iterates of $f^{2}$ to a fixed prime end $E$. Since every principal point of $E$ is fixed under $f^{2} \quad[12$, Lemma 1$]$ and $P(2, f)$ is totally disconnected, $E$ has only one principal point, [14, Corollary, p. 275]. Thus, there is an endcut $A$ of $D_{1}$ which defines $E$. But then, by Lemma $1, f^{2}$ is not recurrent on $\operatorname{Bd}\left(D_{1}\right)$. This contradicts the fact that, since $f$ is p.a.p. on $\operatorname{Bd}\left(D_{1}\right), f^{2}$ is p.a.p. on $\operatorname{Bd}\left(D_{1}\right)$ $[8$, p. 31].

Claim 2 is established.

Since $x \in D_{1}$ and $A_{1}$ is invariant, $f^{2}\left(D_{1}\right)=D_{1}$, hence $B a\left(D_{1}\right) \cup$ $f\left(\operatorname{Bd}\left(D_{1}\right)\right)$ is an invariant subset of $f$.

Claim 3. There is a nondegenerate, invariant subcontinuum $L$ of $A_{1}$ such that if $O$ is the component of $S^{2}-L$ containing $x$, then $p \in \operatorname{Bd}(O)$.

Proof of Claim 3. If $p \in \operatorname{Bd}\left(D_{1}\right)$, then $\operatorname{Bd}\left(D_{1}\right) \cup f\left(\operatorname{Bd}\left(D_{1}\right)\right)$ is the required subcontinuum. Suppose $p \notin \operatorname{Bd}\left(D_{1}\right)$. Let $B_{1}$ be an invariant subcontinuum of 
$A_{1}$ containing $\{p\} \cup \operatorname{Bd}\left(D_{1}\right) \cup f\left(\operatorname{Bd}\left(D_{1}\right)\right)$ and minimal with respect to these properties. Let $V_{2}$ be a ball of radius less than $1 / 2$, centered at $p$. By Lemma 2, there is a set $U_{2} \subset B_{1} \cap V_{2}$, open in $B_{1}$, such that the orbit of $U_{2}$ misses a neighborhood of $\left[B_{1} \cap P(2, f)\right] \cup \operatorname{Bd}\left(D_{1}\right) \cup f\left(\operatorname{Bd}\left(D_{1}\right)\right)$. Let $A_{2}$ be the component of $B_{1}-\operatorname{orbit}\left(U_{2}\right)$ containing $p$. Then $A_{2}$ is invariant, nondegenerate; and, by the minimality of $B_{1}, A_{2}$ does not intersect $\operatorname{Bd}\left(D_{1}\right) \cup f\left(\operatorname{Bd}\left(D_{1}\right)\right)$. If $D_{2}$ is the component of $S^{2}-A_{2}$ containing $\operatorname{Bd}\left(D_{1}\right)$ (and thus containing $\mathrm{Cl}\left(D_{1}\right)$ ), then by the same proof as in Claim 2, $f\left(D_{2}\right) \cap D_{2}=\varnothing$. Also, $f^{2}\left(D_{2}\right)=D_{2}$, since $x \in D_{2}$. And clearly, we must have orbit $\left(U_{2}\right) \cap D_{2} \neq \varnothing$. But then, for some integer $n, f^{n}\left(D_{2}\right) \cap U_{2} \neq \varnothing$. Thus, either $D_{2} \cap U_{2} \neq \varnothing$ or $f\left(D_{2}\right) \cap U_{2} \neq \varnothing$. Hence, $D_{2} \cup f\left(D_{2}\right)$ intersects a neighborhood of $p$ of radius less than $1 / 2$.

If $p \in \operatorname{Bd}\left(D_{2}\right)$, then $\operatorname{Bd}\left(D_{2}\right) \cup f\left(\operatorname{Bd}\left(D_{2}\right)\right)$ is the required subcontinuum. If $p \notin \operatorname{Bd}\left(D_{2}\right)$, we continue the above process. Either we terminate at a finite stage or else we obtain a sequence $D_{1}, D_{2}, \cdots$ of simply-connected domains such that:

(1) $D_{1} \subset \mathrm{Cl}\left(D_{1}\right) \subset D_{2} \subset \mathrm{Cl}\left(D_{2}\right) \subset D_{3} \subset \cdots$;

(2) $\operatorname{Bd}\left(D_{i}\right) \subset A_{1}, f\left(D_{i}\right) \cap D_{i}=\varnothing, f^{2}\left(D_{i}\right)=D_{i}$, for each $i$;

(3) $f\left(D_{i}\right) \cup D_{i}$ intersects a neighborhood of $p$ of radius less than $1 / i$, for each $i$.

If we let $O=\bigcup_{i=1}^{\infty} D_{i}$, and $L=\operatorname{Bd}(O) \cup f(\operatorname{Bd}(O))$, then $L$ is the required subcontinuum, with $p \in \operatorname{Bd}(O) \cap f(\operatorname{Bd}(O))$.

Claim 3 is established.

As in Claim 2, we must have $f(O) \cap O=\varnothing$. And since $x \in O, f^{2}(O)=$ $O$. Now let $L_{1}$ be the outer boundary of $O$ with respect to $f(O)$. Since $p \in \operatorname{Bd}(O) \cap f(\operatorname{Bd}(O))$, we must have $p \in L_{1}$. By Lemma 2, there is a set $V \subset L_{1} \cup f\left(L_{1}\right)$, open in $L_{1} \cup f\left(L_{1}\right)$, such that $\operatorname{orbit}(V)$ misses a neighborhood of $P(2, f) \cap\left[L_{1} \cup f\left(L_{1}\right)\right]$. Let $X$ be the component of $\left[L_{1} \cup f\left(L_{1}\right)\right]-$ orbit $(V)$ containing $p$. Then $X$ is nondegenerate and invariant. Let $W$ be the component of $S^{2}-X$ containing $x$. As before, $f(W) \cap W=\varnothing$. Since $L_{1}-\operatorname{orbit}(V)$ does not separate $x$ and $f(x)$ [13, p. 176], $\mathrm{Bd}(W)$ must contain points of $f\left(L_{1}\right)-L_{1}$. Let $q$ be a point of $\operatorname{Bd}(W) \cap f\left(L_{1}\right), q \neq p$. Since $f\left(L_{1}\right) \subset \operatorname{Bd}(f(O))$, we note that $f(O) \cup\{q\} \cup W$ is a connected set containing $x$ and $f(x)$. Now let $X_{1}$ be an invariant subcontinuum of $X$. containing $p$ and, $\mathrm{Cl}(\operatorname{orbit}(q))$ and minimal with respect to these properties. By Lemma 2, there is a set $Z \subset X_{1}$, open in $X_{1}$, such that orbit( $Z$ ) misses a neighborhood of $\left[P(2, f) \cap X_{1}\right] \cup \mathrm{Cl}(\operatorname{orbit}(q))$. Let $X_{2}$ be the component of $X_{1}-\operatorname{orbit}(Z)$ containing $p$. Then $X_{2}$ is nondegenerate, invariant, and $X_{2}$ misses the set $f(O) \cup\{q\} \cup W$. But then the component of $S^{2}-X_{2}$ containing $f(O) \cup\{q\} \cup W$ is invariant, and we arrive at exactly the same contradiction as in the proof of Claim 2. 
This final contradiction establishes Lemma 3.

LEMma 4. Suppose $f: S^{2} \rightarrow S^{2}$ is a homeomorphism and $U$ is a simply-connected domain such that $f(U) \cap U=\varnothing$ and $f=\operatorname{Id}$ on $\operatorname{Bd}(U)$. Then $\operatorname{Bd}(U)$ is a simple closed curve.

Proof. Fix a point $x \in \operatorname{Bd}(U)$.

Claim. There is an endcut $A$ of $U$ such that $\mathrm{Cl}(A) \cap \mathrm{Bd}(U)=\{x\}$.

Proof of Claim. Let $R_{1}, R_{2}, \cdots$ be a chain of crosscuts defining a prime end whose impression contains $x$, and let $U_{1} \supset U_{2} \supset \cdots$ be the corresponding subdomains of $U$ (so $x \in \bigcap_{i=1}^{\infty} \mathrm{Cl}\left(U_{i}\right)$ ). For each $i$, let $J_{i}$ be the simple closed curve $\mathrm{Cl}\left(R_{i}\right) \cup f\left(\mathrm{Cl}\left(R_{i}\right)\right)$, and let $D_{i}$ be the component of $S^{2}-J_{i}$ containing $U_{i}$. Then $\mathrm{Cl}\left(D_{1}\right) \supset \mathrm{Cl}\left(D_{2}\right) \supset \cdots$. Since the diameters of the $R_{i}$ 's converge to zero, the diameters of the $J_{i}$ 's converge to zero, and so $\bigcap_{i=1}^{\infty} \mathrm{Cl}\left(U_{i}\right) \subset \bigcap_{i=1}^{\infty} \mathrm{Cl}\left(D_{i}\right)$ is the single point $x$. Thus, if $A$ is a half-open arc in $U$ such that each $R_{i}$ separates the endpoint of $A$ from some terminal portion of $A$, we see that $\mathrm{Cl}(A)-A \subset \bigcap_{i=1}^{\infty} \mathrm{Cl}\left(U_{i}\right)=\{x\}$. Thus, $A$ is the required endcut and the claim is proved.

By $[16$, p. 58], $\operatorname{Bd}(U)$ is a simple closed curve provided any two points of $\operatorname{Bd}(U)$ separate $\operatorname{Bd}(U)$. Let $x, y$ be any two points of $\operatorname{Bd}(U)$. By the claim there is a crosscut $A$ of $U$ such that $\mathrm{Cl}(A) \cap \mathrm{Bd}(U)=\{x, y\}$. Then $J=\mathrm{Cl}(A) \cup f(\mathrm{Cl}(A))$ is a simple closed curve, and $\operatorname{Bd}(U)$ must intersect both components of $S^{2}-J$ (otherwise $A$ and $f(A)$ would lie in the same component of $\left.S^{2}-\operatorname{Bd}(U)\right)$. But then $J$ separates $\operatorname{Bd}(U)-\{x, y\}$ in $S^{2}$, hence $\{x, y\}$ separates $\operatorname{Bd}(U)$. The proof of Lemma 4 is complete.

Lemma 5. Suppose $f: S^{2} \rightarrow S^{2}$ is an orientation-reversing, p.a.p. homeomorphism, $K$ is a component of the set of period two points of $f$ such that $K$ contains a fixed point, and $U$ is a component of $S^{2}-K$. Then $f(U) \cap U=\varnothing$, and $f^{2}(U)=U$.

Proof. First we show that $f(U) \cap U=\varnothing$. Suppose not. Then, since $K$ is invariant, $f(U)=U$. Let $G$ be the (upper semicontinuous) decomposition of $S^{2}$ whose only nondegenerate element is $S^{2}-U$. Then the decomposition space $S^{2} / G$ is homeomorphic to $S^{2}\left[16,(2.1)^{\prime}\right.$, p. 171] , and the induced map $g=\pi f \pi^{-1}: S^{2} / G \rightarrow S^{2} / G$ (where $\pi: S^{2} \rightarrow S^{2} / G$ is the decomposition map) is easily seen to be a p.a.p. orientation-reversing homeomorphism. If we denote the set of period two points of $f$ by $P(2, f)$, then $\pi(P(2, f))=$ $P(2, g)$, and $\pi\left(S^{2}-U\right)$ is a degenerate component of $P(2, g)$. This contradicts Lemma 3. Hence $f(U) \cap U=\varnothing$.

Now suppose $f^{2}(U) \neq U$. Then $f^{2}(U) \cap U=\varnothing$. And $f^{2}=$ Id on $\operatorname{Bd}(U)$, since $K \subset P(2, f)$. Hence, by Lemma $4, \operatorname{Bd}(U)$ is a simple closed 
curve. But then $U$ must be one component of $S^{2}-\operatorname{Bd}(U)$ and $f^{2}(U)$ must be the other component. This is impossible because $f^{2}$ is orientation-preserving. Hence $f^{2}(U)=U$, and the proof of Lemma 5 is complete.

THEOREM 6. Suppose $f: S^{2} \rightarrow S^{2}$ is a p.a.p. orientation-reversing homeomorphism which has a fixed point. Then the set of fixed points of $f$ is a simple closed curve.

Proof. Let $K$ be a component of the set of period two points of $f$ such that $K$ contains a fixed point of $f$. Let $V_{1}, V_{2}, \cdots$ be a list of the components of $S^{2}-K$. By Lemma $5, f\left(V_{i}\right) \cap V_{i}=\varnothing$ and $f^{2}\left(V_{i}\right)=V_{i}$, for each $i$. Let $A_{1}=V_{1}$ and $B_{1}=f\left(V_{1}\right)$, and suppose we have defined sets $A_{n}, B_{n}$ which are unions of components of $S^{2}-K$ such that:

(1) $A_{n} \cap B_{n}=\varnothing$;

(2) $V_{1} \cup V_{2} \cup \cdots \cup V_{n} \subset A_{n} \cup B_{n}$;

(3) for each $i, V_{i}$ intersects $A_{n}$ if and only if $f\left(V_{i}\right)$ intersects $B_{n}$.

Form $A_{n+1}, B_{n+1}$ as follows: if $V_{n+1}$ intersects $A_{n} \cup B_{n}$, let $A_{n+1}=$ $A_{n}$ and $B_{n+1}=B_{n}$; if $V_{n+1}$ does not intersect $A_{n} \cup B_{n}$, let $A_{n+1}=$ $A_{n} \cup V_{n+1}$, and $B_{n+1}=B_{n} \cup f\left(V_{n+1}\right)$.

Let $A=\bigcup_{n=1}^{\infty} A_{n}$ and $B=\bigcup_{n=1}^{\infty} B_{n}$. Then $A \cap B=\varnothing, f(A)=B$, $f(B)=A$, and $S^{2}=A \cup B \cup K$.

Define a map $g: S^{2} \rightarrow S^{2}$ by

$$
g(x)= \begin{cases}f(x)\left(=f^{-1}(x)\right), & \text { if } x \in K, \\ f(x), & \text { if } x \in A, \\ f^{-1}(x), & \text { if } x \in B .\end{cases}
$$

It is easily checked that $g$ is a periodic, orientation-reversing homeomorphism, and that the set of fixed points of $g$ is identical with the set of fixed points of $f$. The set of fixed points of $g$ is a simple closed curve by [5]. The proof of Theorem 6 is complete.

6. Orientation-preserving homeomorphisms. In the orientation-preserving case, the similarity between the fixed point sets of p.a.p. homeomorphisms and the fixed point sets of periodic (or weakly almost periodic, see [12]) homeomorphisms no longer holds.

Example. Let $D_{1}, D_{2}, \cdots$ be a (finite or infinite) collection of closed disks in $S^{2}$ such that the union of the $D_{i}$ 's is compact and locally connected, and if $i \neq j$, then $D_{i} \cap D_{j}$ is the south pole $p_{0} \in S^{2}$. For each $i$, let $g_{i}$ be a homeomorphism of $D_{i}$ onto the disk $\left\{(r, \theta) \in R^{2}: r \leqslant 1\right\} \quad((r, \theta)$ polar coordinates). Define $g: S^{2} \rightarrow S^{2}$ by setting $g=$ Id outside the union of the $D_{i}$ 's and setting $g \mid D_{i}=g_{i}^{-1} f g_{i}$ where $f(r, \theta)=(r, \theta+1-r)$. Then $g$ is 
orientation-preserving and p.a.p., and the number of isolated fixed points of $g$ is equal to the number of disks $D_{i}$.

We do, however, have the following partial result.

THEOREM 7. SUppose $f: S^{2} \rightarrow S^{2}$ is a recurrent, orientation-preserving homeomorphism with a finite number of fixed points. Then $f$ has exactly two fixed points.

Proof. The proof consists mostly of combining known results. Let $p$ be a fixed point of $f$, and let $U$ be a neighborhood of $p$ which contains no other fixed points.

Claim. The fixed point index $i(f, U)$ of $f$ on $U$ is equal to +1 (see $[12, \S 4]$ for a short discussion of the local fixed point index $i(f, U)$ or [4] for a more comprehensive treatment).

Proof of Claim. We may assume $U \neq S^{2}$ so that $U$ may be identified with a subset of the plane $R^{2}$. Using the construction in the proof of [6, Lemma 1], we obtain an orientation-preserving homeomorphism $h: R^{2} \rightarrow R^{2}$ such that $p$ is the only fixed point of $h$, and $h=f$ on some neighborhood $V \subset U$, with $p \in V$. (In the proof of Lemma 1 of [6], choose the set $D_{1}$ of that proof to be any neighborhood of $p$ such that $\mathrm{Cl}\left(D_{1}\right)$ is a disk contained in $U$. The construction then easily yields the required homeomorphism $h$.) Since no point of $V$ converges to $p$ under positive or negative iterates of $h$, there is a point $x \in V-\{p\}$ whose orbit under $h$ is contained in $V[8,10.28$, p. 85$]$. If $x$ is not a period two point, the construction given in $\left[10\right.$, p. 89] or $\left[2\right.$, p. 45], yields an arc $A$ in $R^{2}$ (a so-called translation arc) such that $x \in A$, one of the endpoints of $A$ is the image under $h$ of the other endpoint, and $A \cap h(A)$ is this common endpoint. Since $h$ is recurrent at $x$ it is easy to see that $A$ can be chosen so that $h^{n}(x) \in A$ for some $n>1$. If $x$ has period two then the construction of [10, p. 89] yields either a translation arc as in the previous sentence or an arc $A$ joining $x$ and $h(x)$ such that $A \cap h(A)=\{x, h(x)\}$. If $A \cap h(A)=\{x, h(x)\}$, let $J$ be the simple closed curve $A \cup h(A)$. In the case $A \cap h(A)$ is a single point, let $J$ be a simple closed curve constructed from $A \cup h(A) \cup \cdots \cup h^{n}(A)$, where $n$ is the least integer greater than one such that $A \cap h^{n}(A) \neq \varnothing$. Then if $D$ is the bounded component of $R^{2}-J, D$ must contain a fixed point of $h$ [10, Lemma 1.1, p. 89 and Proof of Theorem 1, p. 90]. Thus, $p \in D$. And in fact, the fixed point index $i(h, D)$ is equal to 1 , for, Lemma 1.1 of $[10$, p. 89], shows that as a point $t$ makes one positive circuit of $J$, the vector from $t$ to $h(t)$ turns through an angle of $2 \pi$; thus, the map from $J$ to the unit circle which takes $t$ to $(h(t)-t) /|h(t)-t|$ has degree 1 ; thus, the fundamental class of $H_{2}(D, D-\{p\})$ is mapped to the fundamental 
class of $H_{2}\left(R^{2}, R^{2}-\{0\}\right)$ by the homomorphism induced by Id $-h$, and thus $i(h, D)=1[4]$. But $D-V, V-D$ contain no fixed point of $h$, and $U-V$ contains no fixed point of $f$, hence:

$$
1=i(h, D)=i(h, V)=i(f, V)=i(f, U) .
$$

The claim is established.

Thus, if $U_{1}, \cdots, U_{m}$ is a collection of pairwise disjoint open subsets of $S^{2}$ whose union contains all fixed points of $f$ and such that each $U_{i}, 1 \leqslant$ $i \leqslant m$, contains exactly one fixed point of $f$, then:

$$
\sum_{j=1}^{m} i\left(f, U_{j}\right)=L(f)
$$

where $L(f)$ is the Lefschetz number of $f[12, \S 4]$. But $L(f)=2$, since $f$ is an orientation-preserving homeomorphism. By our claim, $i\left(f, U_{j}\right)=1$, $1 \leqslant j \leqslant m$, hence $m=2$, and the proof of Theorem 7 is complete.

We conclude with two questions.

Question 1. Is there an example of a homeomorphism $f: S^{2} \rightarrow S^{2}$ such that $f$ is recurrent but not p.a.p.?

Question 2. Suppose $f: S^{2} \rightarrow S^{2}$ is an orientation-preserving p.a.p. homeomorphism such that no component of the set of fixed points separates $S^{2}$. Must the set of fixed points have exactly two components?

\section{BIBLIOGRAPHY}

1. R. H. Bing, Concerning hereditarily indecomposable continua, Pacific J. Math. 1 (1951), 43-51. MR 13, 265.

2. L. E. J. Brouwer, Beweis des ebenen Translationsatzes, Math. Ann. 72 (1912), 37-54.

3. M. Cartwright and J. Littlewood, Some fixed point theorems, Ann. of Math. (2) 54 (1951), 1-37. MR 13, 148.

4. A. Dold, Fixed point index and fixed point theorem for Euclidean neighborhood retracts, Topology 4 (1965), 1-8. MR 33 \#1850.

5. S. Eilenberg, Sur les transformations périodiques de la surface de sphère, Fund. Math. 22 (1934), 28-41.

6. O. H. Hamilton, A short proof of the Cartwright-Littlewood fixed point theorem, Canad. J. Math. 6 (1954), 522-524. MR 16, 276.

7. J. G. Hocking and G. S. Young, Topology, Addison-Wesley, Reading, Mass., 1961. MR 23 \#A2857.

8. W. H. Gottschalk and G. A. Hedlund, Topological dynamics, Amer. Math. Soc. Colloq. Publ., vol. 36, Amer. Math. Soc., Providence, R. I., 1955. MR 17, 650.

9. B. de Kerékjártó, Über die periodischen.Transformationen der Kreisscheibe und der Kugelflä̈ne, Math. Ann. 80 (1919-1920), 36-38.

10. - The plane translation theorem of Brouwer and the last geometric theorem of Poincaré, Acta Sci. Math. (Szeged) 4 (1928), 86-102.

11. - Topologische Charakterisienung der linearen Abbildungen, Acta Sci. Math. (Szeged) 6 (1934), 235-262.

12. W. K. Mason, Weakly almost periodic homeomorphisms of the two-sphere, Pacific J. Math. 48 (1973), 185-196. 
13. R. L. Moore, Foundations of point set topology, Amer. Math. Soc. Colloq. Publ., vol. 13, Amer. Math. Soc., Providence, R. I., 1962.

14. M. Ohtsuka, Dirichlet problem, extremal length and prime ends, Van Nostrand, New York, 1970.

15. H. D. Ursell and L. C. Young, Remarks on the theory of prime ends, Mem. Amer. Math. Soc. No. 3 (1951). MR 13, 55.

16. G. T. Whyburn, Analytic topology, Amer. Math. Soc. Colloq. Publ., vol. 28, Amer. Math. Soc., Providence, R. I., 1963. MR 32 \#425.

DEPARTMENT OF MATHEMATICS, RUTGERS UNIVERSITY, NEW BRUNSWICK, NEW JERSEY 08903

Current address: Box 316, Hornitos, California 95325 